\title{
Is Gesture-Speech Mismatch A General Index of Transitional Knowledge?
}

\author{
Michelle Perry
}

University of Michigan

R. Breckinridge Church

Northeastern Illinois University

Susan Goldin-Meadow

The University of Chicago

\begin{abstract}
When asked to explain their beliefs about a concept, some children produce gestures that convey different information from the information conveyed in their speech (i.e., gesture-speech mismatches). Moreover, it is precisely the children who produce a large proportion of gesture-speech mismatches in their explanations of a concept who are particularly "ready" to benefit from instruction in that concept, and thus may be considered to be in a transitional state with respect to the concept. Church and Goldin-Meadow (1986) and Perry, Church and GoldinMeadow (1988) studied this phenomenon with respect to two different concepts at two different ages and found that gesture-speech mismatch reliability predicts readiness to learn in both domains. In an attempt to test further the generality of gesture-speech mismatch as an index of transitional knowledge, Stone, Webb, and Mahootian (1991) explored this phenomenon in a group of 15-year-olds working on a problem-solving task. On this task, however, gesture-speech mismatch was not found to predict transitional knowledge. We present here a theoretical framework, which makes it clear why we expect gesture-speech mismatch to be a general index of transitional knowledge, and then use this framework to motivate our methodological practices for establishing gesture-speech mismatch as a predictor of transitional knowledge. Finally, we present evidence suggesting that, if these practices had been used by Stone et al., they too would have found that gesture-speech mismatch predicts transitional knowledge.
\end{abstract}

This research was supported by Grant \#RO1 HD18617 from the National Institutc of Child Health and Human Development to Susan Goldin-Meadow. We thank Susan Gelman and Jim Stigler for their insightful comments on the manuscript.

Correspondence and requests for reprints should be sent to Michelle Perry, University of Michigan, 3433 Mason Hall, Ann Arbor, MI 48109-1027, or R. Breckinridge Church, Northeastem Illinois University, Department of Psychology, 5500 N. St. Louis Ave., Chicago, IL 60625, or Susan Goldin-Meadow, University of Chicago, Department of Psychology, 5730 S. Woodlawn Ave., Chicago, IL 60637. 
Traditionally, the aim of developmental psychologists has been to describe the stages through which children progress as they acquire new concepts. Increasingly, however, researchers in both developmental and cognitive psychology have begun to focus on characterizing the mental transition that occurs when new concepts are acquired, and describing the mechanisms responsible for transitions of this sort (e.g., Sternberg, 1984).

We have suggested that the relationship between gesture and speech may be a useful tool in characterizing the stability of knowledge states and in predicting the transitions between them. We have discovered that, when asked to explain their beliefs about a concept, some children produce gestures that convey different information from the information conveyed in their speech (i.e., gesturespeech mismatches). These children thus appear to be expressing two different notions with respect to a single concept-one conveyed in speech and one conveyed in gesture. We have shown that those children who produce a large proportion of gesture-specch mismatches in their explanations of a concept (children whom we call "discordant") are more likely to benefit from instruction in that concept than the children who produce few gesture-speech mismatches (children whom we call "concordant"). We have studied this phenomenon with respect to two different concepts at two different ages [conservation in 5- to 8year-olds (Church \& Goldin-Meadow, 1986) and mathematical equivalence in 9to 10-year-olds (Perry, Church, \& Goldin-Meadow, 1988)] and have found, in both domains, that gesture-speech mismatch reliably predicts readiness to learn. Thus, children who are discordant with respect to a particular concept appear to be "ready" to profit from instruction in that concept and, in this sense, can be said to be in a transitional state in their acquisition of the concept.

Moreover, we have shown that the discordant state is transitional, not only in terms of readiness to learn, but also in the sense that it is both preceded and followed by a concordant state. Wagner, Scott, Church, and Goldin-Meadow (1990), for example, showed that children began their acquisition of mathematical equivalence in a concordant state (albeit an incorrect one), then proceeded through a period of discordance, and finally returned to a concordant (and correct) state. In addition, we have shown that there is a cost to being in transition. Goldin-Meadow, Nusbaum, and Garber (1991) reported that children who are in transition with respect to mathematical equivalence (discordant children) expend more cognitive effort when solving addition problems instantiating that concept than children who are not in transition (concordant children) and, as a result, are less capable of simultaneously performing an unrelated word-recall task. We suggest that this increased cognitive cost arises because the discordant children activate, and maintain in working memory, both of the notions found in their mismatched explanations when they solve each of the addition problems.

In an attempt to test the generality of gesture-speech mismatch as an index of transitional knowledge, Stone, Webb, and Mahootian (1991) have explored this phenomenon in a group of 15 -year-olds working on a problem-solving task 
involving multiple causal factors (a modification of Inhelder and Piaget's bending rods task). Confirming our previous studies, Stone et al. found, first, that the adolescent children, when asked to explain their responses on the task, routinely produced iconic gestures along with their speech. In addition, Stone et al. found that these gestures could be reliably coded (even when coded with the sound turned off), and conveyed substantive information about the task. Finally, Stone et al. found that gesture-speech mismatch was quite common, occurring roughly as often as in our own data.

As Stone et al. themselves pointed out, there is no doubt that gesture-speech mismatch exists. It clearly does, even in these older children acquiring a relatively abstract concept. The question, however, is what gesture-speech mismatch reflects and whether its usefulness as an index of transitional knowledge is restricted, either to a small set of concepts or to a narrow range of ages. Stone et al. reported that gesture--speech mismatch did not accurately predict transition in their data, and therefore argued that the measure's usefulness is not as general as we have hypothesized. However, before accepting the limitations of the gesturespeech mismatch index, we need to ask whether Stone et al.'s study replicates the original studies in all aspects. In fact, three components of the Stone et al. study differ from the original studies.

\section{Gesture--Speech Mismatch as an Index of Transitional Knowledge: More Than One Notion in a Single Explanation}

Calculating the Frequency of Gesture-Speech Mismatch. Stone et al. used a different procedure from ours to assess the frequency of gesture-speech mismatch and to divide children into concordant and discordant groups. ${ }^{1}$ The primary difference is that Stone et al. excluded from their calculations all responses in which the child either produced no gestures or produced nonreferential gestures (e.g., batonlike movements that convey no substantive information). We included these responses and offer the following theoretical reason for so doing.

We hypothesize that what characterizes a child in transition with respect to a particular concept is the fact that the child simultaneously activates more than one notion when considering that concept. We are not unique in putting forth this

I Although Stone et al. used a different procedure from ours to calculate the frequency of gesture-speech mismatch, they used the same procedure we did to establish a gestural lexicon. In particular, they determined, in a pilot sample, which gestures routinely accompanied different spoken responses, and took the gesture that most frequently accompanied a particular spoken response to be a lexical match for that response. They then used this lexicon to code the responses of the children in their study, coding gesture with the sound turned off and speech with the picture turned off, as we did in our studies. The fact that such a gestural lexicon can be established is a testament, both to the meticulous and insightful coding performed by Stone et al., and to the systematicity that underlies the gestures individuals produce along with their speech (cf. McNeill, 1992). 
hypothesis. Virtually any theory that posits internal conflict as a mechanism of developmental change assumes that children must, at some level, simultaneously consider and compare the notions they have available in order for discrepancies among those notions to be detected and serve as a catalyst for change (e.g., Keil, 1984; Klahr, 1984; Piaget, 1975/1985). Discordant children exhibit this simultaneous activation of more than one notion quite directly in their explanationsconveying one notion in speech and a second notion in gesture. ${ }^{2}$ Moreover, we have shown that this simultaneous activation of more than one notion is evident, not only in discordant children's explanations of a concept, but also in their solutions to problems instantiating that concept (Goldin-Meadow et al., 1991).

Given that the production of more than one notion characterizes a child in transition, it follows that the production of a single notion ought to characterize the child in a nontransitional state, whether that single notion is produced in both gesture and speech, or in speech alone. By eliminating all of the responses without gesture, as well as those containing nonreferential gestures (which do not reflect substantive notions), Stone et al. have eliminated a large number of the single-notion responses. As a result, their procedure inevitably inflates the proportion of a child's gesture-speech mismatches, and may provide an inaccurate assessment of the relative number of dual-notion responses the child producesthe defining characteristic of discordant versus concordant children. Indeed, Stone et al. did find a higher proportion of discordant children (53\%) than did Perry et al. (35\%) or Church and Goldin-Meadow (46\%), suggesting that Stone et al. may have misclassified as discordant some children who would otherwise have been considered concordant.

A Proposed Reanalysis of the Stone et al. Data. There is, in fact, some suggestion that recalculating the frequency of gesture-speech mismatch to include no-gesture and nonreferential gesture responses would alter the results in the Stone et al. study. Stone et al. divided the children in their study into three groups: spontaneous users who performed well on the task both before and after an intervening training activity, transitional children who performed well on the task after training but not before, ${ }^{3}$ and nonusers who performed poorly on the

2 We do not mean to suggest that the discordant representations in gesture and speech are themselves catalysts for change, but rather that the discordance reflects some discrepancy in the child's representations of the concept, a discrepancy that must be resolved in order for change to occur.

${ }^{3}$ Stone et al. also included in their transitional group one child who succeeded on the task before training but failed on the task after training. We would not have considered such a child to be transitional simply because the standard we use to validate gesture-speech mismatch as an index of transitional knowledge is readiness to learn, and this child had not demonstrated that he or she was ready to learn the concept; see Beilin (1965), Brainerd (1972), Langer and Strauss (1972), Murray (1974), Strauss and Langer (1970), and Strauss and Rimalt (1974) who similarly use receptivity to instruction as the defining characteristic of transitional knowledge. 
task both before and after training. They predicted that the spontaneous users (assumed to be stable in their mastery of the concept) and the nonusers (assumed to be stable in their lack of mastery of the concept) would produce fewer gesturespeech mismatches before training than the transitional children (assumed to be on the verge of learning the concept).

Stone et al. found that the spontaneous users produced gesture-speech mismatches on $32.4 \%$ of their tests, which contained both gesture and speech prior to training, a percentage that was not significantly different from the percentage produced by either the transitional children or the nonusers. It is important to note, however, that the spontaneous users produced significantly more tests without gesture or with nonreferential gestures than the transitional children or the nonusers. If these tests are included in the denominator when the percentage of tests containing gesture-speech mismatch is calculated, it follows that the percentage of gesture-speech mismatch would decrease for the spontaneous users, but not for the transitional children or the nonusers-an outcome that might result in spontaneous users having significantly fewer tests with gesturespeech mismatch than the transitional children or the nonusers. Thus, recalculating the frequency of gesture-speech mismatch to include no-gesture and nonreferential gesture responses would lead to an outcome more in line with the predictions Stone et al. made on the basis of our original studies (note, however, that our theory predicts that the nonusers should also produce fewer gesturespeech mismatches than the transitional children; we return to this discrepancy in Section 2 on type of training).

A Reanalysis of the Perry et al. Data. A second argument that supports our claim that the way in which gesture-speech mismatch is calculated is pivotal to exploring the question of transition comes from a reanalysis of our own data. If we recalculate the frequency of gesture-speech mismatch ignoring those responses that did not contain gesture (i.e., using the procedure Stone et al. used), ${ }^{4}$ the pattern of results obtained look very much like those presented in Stone et al. Table 1 (p. 114) presents the proportion of children in Perry et al. who acquired the concept of mathematical equivalence after training (i.e., those who succeeded on both the addition posttest and the multiplication generalization test) as a function of the child's concordance or discordance status on the pretest. Concordance and discordance were calculated twice: once as in the original Perry et al. study, including all responses (even those without gesture), and a second time as in Stone et al., excluding responses that did not contain gesture. To understand

\footnotetext{
${ }^{4}$ Nonreferential gestures were not coded in either Church and Goldin-Meadow (1986) or Perry et al. (1988). As a result, if a verbal response had occurred with a nonreferential gesture but with no other gesture, it would have been considered a speech-alone response in the original analyses, and therefore would have been automatically eliminated along with the other speech-alone responses in the reanalyses following the Stone et al. procedure in Tables 1 and 2.
} 


\section{Table 1. Proportion of Concordant and Discordant Children Succeeding on the Generalization Test After Training}

\begin{tabular}{lcc}
\hline $\begin{array}{l}\text { Proportion of Children Succeding } \\
\text { on the Generalization Test }\end{array}$ & $\begin{array}{c}\text { Concordant } \\
(n)\end{array}$ & $\begin{array}{c}\text { Discordant } \\
(n)\end{array}$ \\
\hline
\end{tabular}

If Concordance und Discordance are Calculated:

1. As in Perry et al., i.e., including all explanations $.25(24)^{b}$ $.62(1.3)^{*}$

2. As in Stone et al., i.e., including only those explanations containing both gesture and speech

aConcordant children were defined as those who produced gesture-speech mismatches on fewer than $50 \%$ of the countable explanations they produced on the pretest (i.e., all of the explanations they produced for the Perry et al. analyses, and the gesture + speech explanations they produced for the Stone et al. analyses). Discordant children were defined as those who produced gesture-speech mismatches on 50\% or more of the countable explanations they produced on the pretest.

bThe number in parentheses represents the total number of concordant children or discordant children contributing data to each analysis. The reason that the number of subjects differs for the Perry et al. and the Stone et al. analyses is because 7 of the children in the study produced no gestures at all. Because Stone et al. considered only those responses that contained both gesture and speech as part of the data base, these children provided no data for the Stone et al. analysis. In contrast, because Perry et al. included all responses-even those without gesture-as part of the data base, these 7 subjects can be included in the Perry et al. analysis. Following the Perry et al. procedures, the 7 children would be elassified as concordant because fewer than 50\% (in fact, none) of their 6 explanations contained gesture-specch mismatch (i.e., all of their responses contained a single notion). It is worth noting that if these 7 children were included as concordant in the Stone et al. calculations, the proportion of children succeeding on the generalization test would still not be significantly different for the concordant and discordant children.

${ }^{*} p<.05$.

the effect of such a recalculation, consider a child who produced two gesturespeech mismatches, one gesture-speech match, and three speech-alone responses in his six explanations on the pretest. Following Perry et al., the child would be considered concordant: He produced two mismatches out of his six responses, that is, $33 \%$ of his responses are mismatches, a percentage lower than the $50 \%$ needed to be classified as discordant. However, following Stone et al., the same child would be considered discordant: He produced two mismatches out of three responses (only those containing gesture count toward the denominator), that is, $67 \%$ of his responses are mismatches, a percentage greater than the $50 \%$ needed to be classified as discordant.

We have hypothesized that children who are discordant with respect to a concept are more likely to benefit from instruction in that concept than children 
Table 2. Average Proportion of Explanations Containing a Gesture-Speech Mismatch Produced by Transitional and Nontransitional Children on the Pretest

\begin{tabular}{|c|c|c|}
\hline $\begin{array}{l}\text { Average Proportion of } \\
\text { Explanations Containing } \\
\text { Gesture-Speech Mismatch }\end{array}$ & $\begin{array}{l}\text { Nontransitional } \\
\qquad M(S D)\end{array}$ & $\begin{array}{c}\text { Transitional }^{\mathrm{A}} \\
M(S D)\end{array}$ \\
\hline $\begin{array}{l}\text { If the Total Number of Explan } \\
\text { 1. As in Perry et al., i.e., in- } \\
\text { cluding all explanations } \\
\text { 2. As in Stone et al., i.e., in- } \\
\text { cluding only those explana- } \\
\text { tions containing both gesture } \\
\text { and speech }\end{array}$ & $\begin{array}{l}\text { IS is Calculated: } \\
.25(.29)\end{array}$ & $.42(.26)^{*}$ \\
\hline
\end{tabular}

"Nontransitional children were defined as those who did not succeed on the generalization test after training. Transitional children were defined as those who did succeed on the generalization test after training.

${ }^{*} p<.05$.

who are concordant. Note, however, that in Table 1 this prediction is confirmed only if the frequency of gesture-speech mismatch is calculated on the basis of all of the child's responses, even those without gesture. That is, discordant children are more likely to succeed on the generalization test than concordant children only if discordance is calculated according to Perry et al. $\left[\chi^{2}(1)=4.79, p=\right.$ .03], and not if discordance is calculated according to Stone et al. $\left[\chi^{2}(1)=.62\right.$, $p=.43] .5$

In their study, Stone et al. also looked at the relationship between gesturespeech mismatch and transitional status by dividing children into transitional and nontransitional groups and calculating the average proportion of gesture-speech mismatches the groups produced. Following the line of reasoning developed in our studies, they predicted that the transitional children would produce more gesture-speech mismatches prior to training than the nontransitional children (although they found no evidence for this prediction in their data). Table 2 presents a reanalysis of the Perry et al. data dividing the children into a transitional group (those who succeeded on the generalization test after training) and a nontransitional group (those who failed the generalization test after training), and

${ }^{5}$ It is interesting to note that when we consider only the children in Stone et al.'s study who did not know the concept at the beginning of the study (i.e, the nonusers and the transitional children), we find that the proportion of discordant children who succeeded on the task after training was, in fact, slightly but not significantly higher than the proportion of concordant children: .53 (1\%/9) versus .44 (7/10) when calculated following Stone et al.'s procedure. This difference is comparable to the slight difference seen in Table 1 when the Perry et al. data are analyzed according to the Stone et al. procedure: $.53(\% / 17)$ versus $.38(5 / 13)$. 
calculating the mean proportion of responses containing gesture-speech mismatches each group produced prior to training. We have calculated the mean proportion of responses containing gesture-speech mismatch twice: once following the procedure used in Perry et al., including all responses (even those without gesture), and a second time following the procedure used in Stone et al., excluding responses that did not contain gesture. As Stone et al. would predict, transitional children produced significantly more gesture-speech mismatches than nontransitional children. However, this prediction is confirmed only if the frequency of gesture-speech mismatch is calculated according to the procedure used in our original studies $[t(35)=1.71, p<.05]$, and not if the frequency is calculated according to the procedure used in Stone et al. $\mid t(28)=.32, p=$ .37]. ${ }^{6}$

In sum, we have argued on theoretical grounds that all of a child's responses-those without gesture as well as those with gesture-ought to be included when the frequency of gesture-speech mismatch is calculated. We argue specifically that no-gesture responses, by definition, reflect the use of a single notion (expressed only in speech) and thus contrast in an important way with responses in which gesture-speech mismatch reflects the use of more than one notion. Next, we have presented empirical evidence suggesting that, if Stone et al. had included no-gesture responses (and responses with nonreferential gestures) in their own data base, they would have been more likely to find the relationship between gesture-speech mismatch and transitional knowledge predicted by our hypothesis. Finally, we have presented a reanalysis of our own data showing that if we use Stone et al.'s procedure for calculating gesture-speech mismatch, we also can find no relationship between gesture-speech mismatch and transitional knowledge.

\section{Validating Gesture-Speech Mismatch as an Index of Transitional Knowledge: The Importance of the Type of Training Used to Identify Children in Transition}

As previously described, recalculating the frequency of gesture-speech mismatch in the Stone et al. data to include those tests that contained no gestures or nonreferential gestures is likely to result in the spontaneous users producing fewer gesture-speech mismatches than either the transitional children or the nonusers. However, because the nonusers produced approximately the same number of tests without gesture or with nonreferential gesture as the transitional children, a recalculation is not likely to result in the nonusers and transitional

6 The number of subjects used for these two analyses differs because 7 of the subjects in the Perry et al. study did not gesture at all. Because Stone et al. considered only those responses that contain both gesture and speech as part of the data base, these children provide no data for the Stone et al. analyses. In contrast, because Perry et al. included all responses, even those without gesture in the data base, these 7 subjects can be included in this analysis. 
children having different proportions of tests containing gesture-speech mismatch. According to our theory, we would have expected the transitional children (who are presumed to be on the verge of learning the concept) to produce gesture-speech mismatch more frequently than the nonusers (who are presumed to be stable in their ignorance of the concept). However, this prediction depends heavily on whether Stone et al. used a training procedure to stimulate learning that is adequate for truly discriminating transitional from nontransitional children.

It is our belief that, to be considered in transition, a child must be able to respond to instruction by demonstrating more than rote or superficial learning. Indeed, we have proposed gesture-speech mismatch as a technique for identifying precisely those children who are capable of demonstrating qualitative (as opposed to superficial) shifts in their understanding of a concept. We have thus argued that discordant children are particularly ready to profit from instruction in a concept, and experience a qualitative change in their understanding of the concept, as a result of that instruction.

It is important to realize that we are not arguing that discordant children are more susceptible than concordant children to any kind of instruction-only to the type of instruction that leads to qualitative change. Thus, certain kinds of training would not be expected to have differential effects on discordant and concordant children (e.g., training that improves performance on the same task but does not provide skills that can be generalized; see Hiebert \& Lefevre, 1986), and would consequently not be adequate for identifying transitional versus nontransitional children.

For example, our previous work has shown that discordant children are no more likely to progress on the mathematical equivalence task than concordant children when given training in a procedure for solving addition equivalence problems. Figure l(A) (p. 118) presents the proportion of concordant and discordant children who showed no success, success on only the posttest, or success on both the posttest and the generalization test after procedure training (data from Perry, 1987/1988). Procedure training did not distinguish between concordant and discordant children - both groups made approximately the same amount of progress on the posttest, which contained the same type of addition problems as in the pretest $\left[X^{2}(1)=.83, p=.36\right]$, and both groups made essentially no progress on the generalization test, which required that the children extend the knowledge gained during training to a different type of problem $\left[\chi^{2}(1)=1.37, p=.24\right]$.

In contrast, training in the principle underlying mathematical equivalence did lead to success on the multiplication generalization test and, moreover, did have a differential effect on discordant and concordant children. Figure 1(B) (p. 118) presents the proportion of concordant and discordant children who showed no success, success on only the posttest, or success on both the posttest and the generalization test after principle training (data from Perry et al., 1988). Unlike procedure training, principle training had different effects on the two groups, 


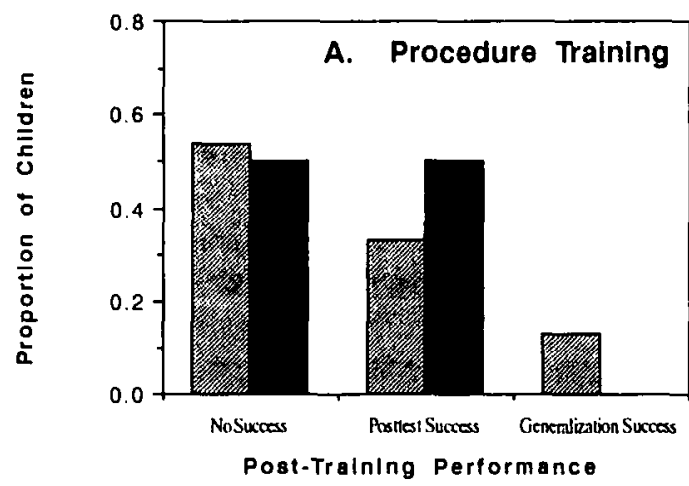

Concordant

Discordant

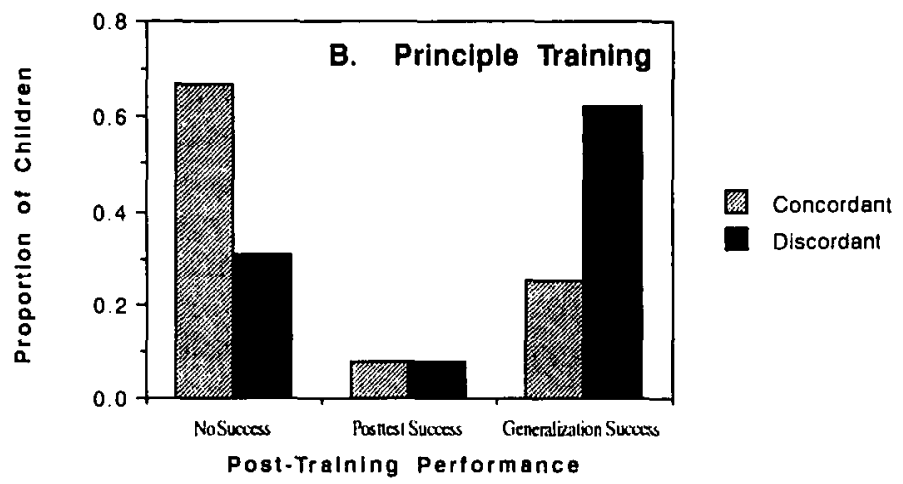

Figure 1. The proportion of concordant and discordant children who showed no success, success on only the posttest, or success on both the posttest and the generalization test under two different training conditions: (A) after training in a procedure for solving addition equivalence problems, or (B) after training in the principle underlying mathematical equivalence. Note that procedure training for the most part does not lead to success on the generalization test and does not distinguish between concordant and discordant children. In contrast, principle training does both.

stimulating the discordant children to do significantly better on the generalization test than the concordant children $\left[\chi^{2}(1)=4.79, p=.03\right]$. Thus, performance after principle training could serve as the basis upon which to identify children in transition, but performance after procedure training could not. The crucial question is whether the type of training Stone et al. used in their study was adequate to identify children in transition.

Stone et al. chose the training for their study from one of several types of interventions shown by Day and Stone (1982) to improve performance in some 
children from one trial of a control-of-variables task to a second trial of the same task. Stone et al. asked children to decide which of two pairs of rods would provide a better way to identify the variables causing the rod to bend (where one of the pairs was a confounded test and the other an unconfounded test). Stone et al. provided no evidence that this type of training is sufficient to lead to a general understanding of how to isolate variables, even for a child who is in transition with respect to this concept; that is, the training may make evident the contrast between confounded and unconfounded tests for the bending rods task but not for other, conceptually related tasks (e.g., testing which combinations of chemicals produce a yellow liquid). ${ }^{7}$ If so, this training would not identify as ready to learn children who are capable of making qualitative shifts in their understanding of the concept (viz. discordant children under procedure training) and therefore, we would argue, should not have been used to attempt to discriminate transitional from nontransitional children.

\section{The Relationship Between Changing Knowledge and Discordance: The Path of Acquisition}

The final point we emphasize is that, in our view, the discordant state is transitional in the sense that it is both preceded and followed by a concordant state; that is, when children acquire a concept, they are first concordant (although incorrect), then discordant, and finally again concordant (and correct) with respect to that concept. The two concordant states are comparable in the sense that children in both of these states ought to produce few gesture-speech mismatches (i.e., primarily single-notion explanations). However, it is crucial to realize that, although the children in the two concordant states are both hypothesized to be stable in their knowledge of the concept, the level of their knowledge is expected to differ markedly. In particular, children in the concordant state that precedes discordance, although stable, are stable in their lack of understanding of the concept; in contrast, children in the concordant state following discordance are stable in their mastery of the concept.

This point is particularly relevant to the analyses Stone et al. presented in their Table 3 where they examined the children's judgments of whether a test was

\footnotetext{
7 Day and Stone (1982) did attempt to examine the children's ability to generalize the knowledge gained during intervention to a different control-of-variables task (the pendulums task), and showed that generalization of this sort did occur. However, they did not present the generalization data as a function of type of intervention. Thus, one cannot tell from the data presented in Day and Stone whether the type of training Stone et al. chose to use in their study was one of the interventions that led to generalization. Moreover, because Day and Stone did not include a pretest measure on the pendulums task, and because there is no a priori reason to believe that the bending rods task ought to be mastered before the pendulums task, it is difficult to tell if the children who did well on the pendulums generalization task did so because of what they learned during training, or because they knew how to do the task before the experiment began.
} 
confounded, and their justifications for those judgments. In this table, Stone et al. contrasted concordant and discordant children, but they included in their sample of concordant children those children who were spontaneous users (i.e., those who knew the task before training and were likely to have been in the concordant state that follows discordance), as well as those children who were nonusers (i.e., those who did not improve on the task even superficially after training and were likely to have been in the concordant state that precedes discordance). These two types of concordant children need to be distinguished and separated in analyses of this type precisely because we would predict that children in the concordant state following discordance ought to perform better than children in the concordant state preceding discordance.

It is worth pointing out that the children in our training studies were selected because they failed the pretest; thus, the only type of concordant child in our studies was the child in the concordant state preceding discordance. We did, however, find in the Perry et al. study that, after training, approximately onc third of the children who were discordant on the pretest became concordant but at the higher, equivalence level (see Table 5 in Perry et al.). We have, in addition, gathered further evidence in support of the path children are hypothesized to follow when acquiring mathematical equivalence. In an extended training study, Wagner et al. (1990) instructed children in mathematical equivalence and observed their explanations throughout the training period. As the children acquired the concept of mathematical equivalence, they progressed through a series of steps: They began acquisition in a concordant state at a nonequivalence level, then proceeded through a period of discordance, and finally returned to a concordant state but at a higher, equivalence level. These data add credence to the hypothesis that the discordant state is transitional, not only in terms of readiness to learn, but also in the sense that it is both preceded and followed by a concordant state.

\section{Summary}

The data presented by Stone et al., taken in conjunction with our own, make it clear that gesture-speech mismatch exists. Indeed, gesture-speech mismatch appears to be relatively common in different age groups acquiring different concepts. The question, however, is whether gesture-speech mismatch is a reliable index of transitional knowledge for individuals of any age acquiring a variety of concepts.

We have provided a theoretical framework that allows us to understand why gesture-speech mismatch, reflecting the use of dual notions, might well be a general index of transitional knowledge, and we have argued for methodological practices in accord with this theoretical position. If these practices are used, gesture-speech mismatch is a reliable predictor of transitional knowledge in at least two concepts acquired at two different ages. In this context, we point to evidence from a laboratory other than our own suggesting that gesture-speech 
mismatch is also a reliable predictor of readiness to learn in very young children acquiring words. Gershkoff-Stowe and Smith (1991) showed that children ranging in age from 16 to 22 months produced a relatively large proportion of gesture-speech mismatches (i.e., pointing-naming errors for known words, such as pointing at a cow while saying "dog" at a time when dog was a reliably known word) during the period when they were experiencing a "naming explosion" and were particularly ready to add new words to their lexicons.

Finally, we argue that if these practices were to be used by Stone et al., that is, if attention were paid to the child's production of single-notion as well as dualnotion responses, and if care were taken to use a training procedure that induces qualitative change to identify children who are in transition, it might well turn out that gesture-speech mismatch would signal readiness to learn in their data as it did in our own and Gershkoff-Stowe and Smith's. If not, the interesting question to ask is why gesture-speech mismatch reflects transitional knowledge in some concepts and not in others-and, equally important, what it is that gesture-speech mismatch does reflect in these other concepts.

\section{REFERENCES}

Beilin, H. (1965). Learning and operational convergence in logical thought development. Journal of Experimental Child Psychology, 2, 317-339.

Brainerd, C.J. (1972). The age-stagc issue in conservation acquisition. Psychonomic Society, 29, $115-117$.

Church, R.B., \& Goldin-Meadow, S. (1986). The mismatch between gesture and speech as an index of transitional knowledge. Cognition, 23, 43-71.

Day, M.C., \& Stone, C.A. (1982). Developmental and individual differences in the use of the control-of-variables strategy. Journal of Educational Psychology, 74, 749-760.

Gershkoff-Stowe, L., \& Smith, L.B. (1991, April). Changes in pointing and labeling during the naming explosion. Poster presented at the biennial meeting of the Society for Research in Child Development, Seattle, WA.

Goldin-Meadow, S., Nusbaum, H., \& Garber, P. (1991, April). Transitions in learning: Evidence for multiple hypotheses. Paper presented as part of a symposium on Transitional Knowledge at the biennial meeting of the Society for Research in Child Development. Seattle, WA.

Hiebert, J., \& Lefevre, P. (1986). Conceptual and procedural knowledge in mathematics: An introductory analysis. In J. Hiebert (Ed.), Conceptual and procedural knowledge: The case of mathematics (pp. 1-27). Hillsdale, NJ: Erlbaum.

Keil, F.C. (1984). Mechanisms of cognitive development and the structure of knowledge. In R.J. Stemberg (Ed.), Mechanisms of cognitive development (pp. 81-99). New York: W.H. Freeman.

Klahr, D. (1984). Transition processes in quantitative development. In R.J. Sternberg (Ed.), Mechanisms of cognitive development (pp. 101-139). New York: W.H. Freenan.

Langer, J., \& Strauss, S. (1972). Appearance, reality and identity. Cognition. I, 105-128.

McNeill, D. (1992). Hand and mind: What gestures reveal about thought. Chicago: The University of Chicago Press.

Murray, J.P. (1974). Social learning and cognitive development: Modelling effects on children's understanding of conservation. British Journal of Psychology, 65, 151-160.

Perry, M. (1988). Transitional knowledge in the acquisition of concepts (Doctoral dissertation, University of Chicago, 1987). Dissertation Abstracts International, 48, 3433. 
Perry, M., Church, R.B. , \& Goldin-Meadow, S. (1988). Transitional knowledge in the acquisition of concepts. Cognitive Development, 3. 359-400.

Piaget, J. (1985). The equilibration of cognitive structures. Chicago: University of Chicago Press. (Original work published 1975)

Sternberg, R.J. (Ed.). (1984). Mechanisms of cognitive development. New York: W.H. Freeman.

Stone, A., Webb, R., \& Mahootian, S. (1991). The generality of gesture-speech mismatch as an index of transitional knowledge: Evidence from a control-of-variables task. Cognitive Development, 6, 301-313.

Strauss, S., \& Langer, J. (1970). Operational thought inducement. Child Development, 40, 163-175.

Strauss, S., \& Rimalt. 1. (1974). Effects of organizational disequilibrium training on structural elaboration. Developmental Psychology, 10, 526-533.

Wagner, M.M., Scott, M., Church, R.B., \& Goldin-Meadow, S. (1990, May). Disequilibrium in concept acquisition: What the hands reveal about a child's state of mind. Paper presented at the 20th Anniversary Symposiun of the Jean Piaget Society, Philadelphia, PA. 